\title{
REMINISCENCES OF EARLY DAYS OF THE VLA
}

\author{
DAVID S. HEESCHEN \\ National Radio Astronomy Observatory, Charlottesville, VA
}

Listening to older folks reminisce has never been one of my favorite pastimes, and I suspect it isn't one of yours either so I've been a little apprehensive about what to say at this affair. I finally decided to say as little as possible and still be able to justify someone paying my way out here. So I'll try to be brief, and I hope relatively painless. However I do have a prepared speech here which I am going to read. I can no longer speak extemporaneously or from notes. Maybe I never could very well, but now I don't even try. This is because my brain, like the universe, has evolved to where it consists primarily of voids and dark matter.

I'd like to give you a short chronological overview of the VLA project, and then talk a bit more about the very early days, that is from about 1961 through 1965 or 66 . I think the following speakers can cover the later period much better than I, and by sticking to the 60's I run less risk of being contradicted, even when I'm wrong.

I have outlined below the VLA chronology. I want to mention just some of the items on this list that I think are particularly interesting or important. Discussions and some design work on what would eventually become the VLA began in 1961 at Green Bank, which at that time was NRAO's only site. One possibly interesting bit of trivia from that era relates to the acronym "VLA." It was originally used at NRAO in the late 50's to mean "Very Large Antenna" because we were interested then in a large diameter dish, and continued to be well into the 60's. It was first used for "Very Large Array" in 1962 as nearly as I can determine by looking back through the files.

\section{VLA Chronology}

1961-1963 Preliminary design work

1961 Report of NSF Advisory Panel on Large Radio Telescopes (NSF 1961)

$1962 \quad$ Phrase 'Very Large Array' came into use

1964 Whitford Committee' report (NAS 1964)

Green Bank interferometer project begun

VLA design group organized (G.W. Swenson, chair)

1964-1967 Intensive design effort

$1965 \quad$ NRAO Users Committee formed

First visit to Plains of San Augustin by NRAO personnel

$1966 \quad$ First VLA report distributed

1967 VLA proposals, Vols. I and II, issued (NRAO 1967)

Plain of San Augustin proposed as site

First 'Dicke Committee' meeting (NSF 1967)

1967-1969 Further design work. Design group disbanded in 1969 
1971 'Greenstein Committee' recommendations established (NAS 1972)

VLA design work recommended

Stanford Research Institute undertook VLA feasibility study for NSF (SRI 1972)

VLA proposal, Vol. IV, issued (NRAO 1971)

1972 Construction project management organized

(J.H. Lancaster, project manager)

VLA approved. First funding received

1973 Antenna contract signed

1974 Site work begun

1975 VLA project personnel move to New Mexico

Two-antenna interferometer operating at site

1976 Ad Hoc Advisory Panel reviews VLA for NSF, Congress

(NSF 1977)

1977 Scientific observing begun, with six antennas

1979 Twenty-eighth antenna completed

1981 VLA construction completed

The design work percolated along in a low-key way until 1963-64, when several important things happened. The Green Bank interferometer project was begun; the "Whitford Committee" published its recommendations; and a VLA design group was more or less formally organized, chaired by George Swenson. The Whitford Committee was the first of the so-called decade reviews of astronomy, and its recommendations included radio astronomy instruments. I'll talk more about all of these in a few minutes. In 1965 Cam Wade made the first visit by NRAO personnel to the Plains of San Agustin. I believe he was accompanied by Sid Smith, but I'm not sure. I think that Bill Erickson was the first to call attention to the Plains as a possible radio astronomy site, but of course it also showed up prominently in the map searches done by Cam Wade. Figure 1 shows a picture of Cam and Max Small at the site.

The first report of the VLA was distributed in 1966. It was about as thick as the present MMA proposal, and the NSF refused to consider it seriously because it was too thin. In those days everything we sent to the NSF was weighed rather than read. But the report did generate a lot of useful discussion, outside the NSF. The design effort was discontinued in 1969 because it had progressed to the point where further work was, or should be, closely tied to the construction, and construction funding didn't look to us to be very promising. This was partly because of the general budget situation and partly because of our interpretation of the second Dicke Committee report. The two Dicke Committee meetings and subsequent reports, in 1967 and 1969 , were called by the NSF to assess and make recommendations regarding the various major radio astronomy proposals that were extant at that time. The first report, in 1967, was generally favorable to the VLA, but it also questioned some aspects of the design and recommended additional work. 


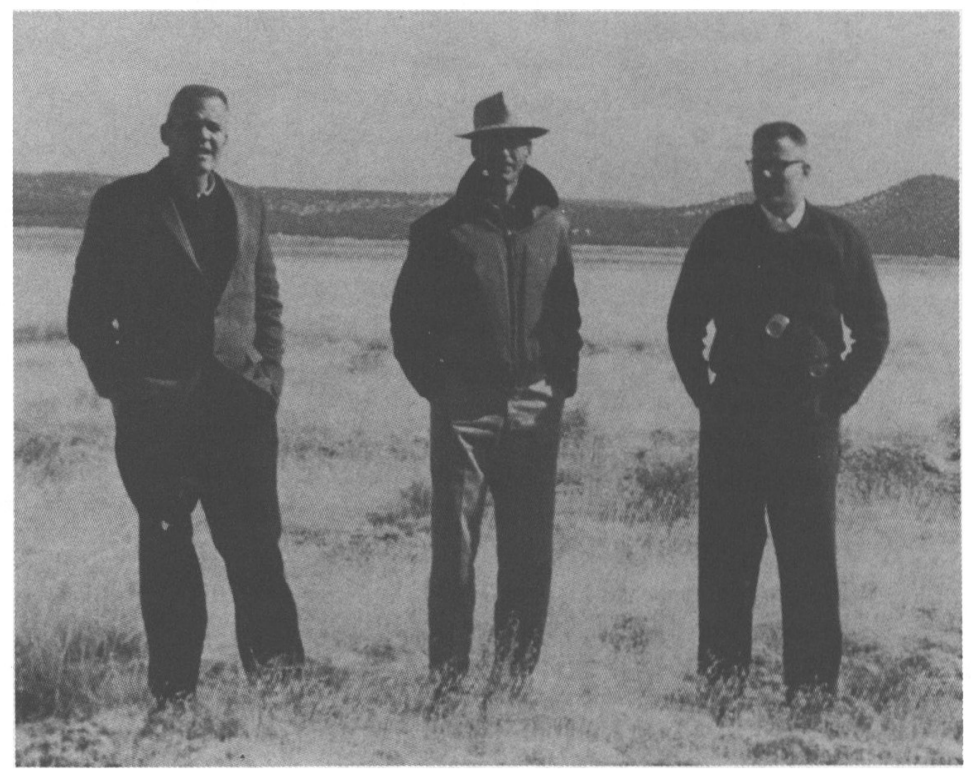

Figure 1. A photograph of Max Small, Bob Weber, and Cam Wade, taken in February 1966 on the Plains of San Augustin by George Swenson.

The second report, in 1969, must have been somewhat ambiguously written, because different people read different things between the lines. It seemed to me to damn the VLA with faint praise and was widely regarded both in and outside of NRAO as sounding the death knell of the project. Later, I spoke individually to each member of the committee and each claimed that that was not their intent. In any case, this was the nadir of the VLA project as far as I was concerned. A few years later, however, the VLA was alive again, strongly endorsed by the Greenstein Committee and a whole host of other committees. So the report did not have the effect we thought it would have, and the Dicke Committee members all became "good guys" again in my eyes.

The Greenstein Committee, you will remember, was the second "decade review" of astronomy, following the Whitford Committee. I am often asked whether I think the Greenstein Committee endorsement was essential to getting the VLA funded, and I don't really know the answer. I suspect that if the Greenstein Committee had never existed the VLA would still have been funded, but once the committee was created, its endorsement of the VLA was probably essential.

The project was revived again in the spring of 1971 , because of various indications that it would finally be funded. The design was dusted off and revised under the general direction of Hein Hvatum. It is worth noting that the VLA design of 1971-72 was significantly different from that of 1969, partly because new technology allowed different and better designs and partly because during the 1969-71 interim when design work was formally in abeyance a lot 
of developments took place at Green Bank, mostly by Sandy Weinreb and his group, that were then incorporated into the VLA. For example, the 4-feed system developed for the $140-\mathrm{ft}$ in this period became in effect the prototype of the VLA frontend system, and it was very different from the 1969 design. So while the VLA didn't change in concept or in its major goals, it did change considerably in detail, and very much for the better. The early design work was in no sense a waste of effort however. It showed that the VLA would work, and was the basis for its acceptance by the funding agency and the community, so it was absolutely essential.

Jack Lancaster joined us as project manager in 1972, and in November of 72 (that is the beginning of FY 73) construction funds were made available, and the previous eleven years of toil and trouble finally began to amount to something. We still had some problems ahead of us; for example, the mid-east war, dollar devaluation, and period of rapid inflation which threatened to blow our budget, but Jack in fact brought the project to a successful conclusion on time and within the budget. A key contract, for 28 antennas, was signed in 73 , site work began in 74 , a 2 - antenna interferometer was operating at the site in 75 , scientific observing, with six antennas, began in 77 , and the job was formally completed in 1981 . Since then of course there have been major improvements to the instrument, especially in the areas of computers and receivers, and it is now a vastly more powerful telescope system than it was even in 1981. That's a separate story, in which I played no part. Those who did deserve a great deal of credit.

The specific improvements that have occurred, and that make the VLA so much more powerful, were of course not anticipated in 1961 when the project began, but it was anticipated very early that some evolution eventually would be desirable, and one of the very early goals of the design was for a system that would be able to adapt to the future. We didn't want to design a dinosaur. So now I'd like to go back and say a little about the early stages of the VLA design, from 1961 to about 65 or 66 .

Things were different in 1961, so different in fact that it is a little hard to appreciate today the climate in which the VLA was developed. As I look at all the youthful faces in front of me I'm tempted to subtitle these reminiscences "Tales From the Dark Ages." There was only one radio spectral line, no quasars, pulsars, radio stars, plerions, no mag tapes, pc's, e-mail, no CLEAN or self-cal. Major instruments included the Mills Cross and Cambridge arrays, the 250-ft at Jodrell Bank, and the Parkes 210- $\mathrm{ft}$. In the US, the $140-\mathrm{ft}$, $300-\mathrm{ft}$, and Arecibo telescopes were under construction, there were several $60-85 \mathrm{ft}$ antennas, and the Caltech interferometer of two $90-\mathrm{ft}$ dishes. Note too that telescope diameters were still measured in feet rather than meters. Enormous effort was going into collecting and improving data-catalogs of sources, positions, identifications, source sizes and structures. In the case of identifications, in 1962 radio sources had been identified with a few tens of SNR and peculiar galaxies, and a few more tens of HII regions and normal galaxies. John Bolton's interferometer at Owens Valley had about 2 arcmin resolution, while Henry Palmer's radio link interferometer at Jodrell Bank had about 3 arcsec resolution; but with these, and all other interferometers, only a very few spacings were measured. Lots of Galactic $21 \mathrm{~cm}$ line work was being done at Harvard, Jodrell Bank, The Netherlands, and Australia. It all sounds primitive by today's standards, but in fact it was an exciting and stimulating 
time and new developments came thick and fast, mostly from Australia and England.

In spite of the great advances that had been made, existing telescopes of the 60 's had some clear limitations:

- angular resolution was poor

- imaging ability was non existent or very slow

- interferometers and arrays had complex side lobe/grating lobe patterns that complicated interpretation of observations

- almost all interferometers were one dimensional-sky coverage was poor

- there was generally a big imbalance between confusion and sensitivity limits-most instruments were limited far too soon by one and therefore not able to make full use of the other

- the telescopes were very slow

These instrumental drawbacks led to some fundamental problems in both the collection and the interpretation of observations. For example, for a long time there were vigorous arguments about $\log \mathrm{N}-\log \mathrm{S}$. Were this person's results biased by grating or side lobe confusion, or that person's by counting groups of sources as a single source? Another problem was that the dynamic range of the phenomena being studied-luminosity, dimensions, etc.far exceeded the dynamic range of any given instrument. We were trying to observe a broad band universe with a set of extremely narrow band filters, and the resultant selection effects were severe. That, and the slowness of gathering data, meant that each source was, in effect, unique. It was pretty hard to find what, if anything, was typical and what was not.

So that's my very subjective, thumbnail sketch of radio astronomy in the early 60 's. There were many scientific challenges, but the most exciting and challenging seemed to be extragalactic sources. That, and the perceived instrumental limitations I just listed, pretty much shaped the design of the VLA. One other aspect of the 60's influenced the VLA design, and that was our concept of the NRAO as a national center. Today that concept is wellestablished and pretty much taken for granted. That was not the case in the 60 's. There was a more or less continuous and sometimes bitter debate about what NRAO should be, and whether it should even exist at all.

As I said, the first discussions about what eventually became the VLA began at Green Bank in 1961. The people involved initially were Frank Drake, John Findlay, Dave Hogg, Hein Hvatum, Ivan Pauliny Toth, V. R. Venugopal, Mark Vinokur, Campbell Wade, and me. Most radio astronomy in those days was done by radio physicists and engineers who invented, built, and then used their own instruments. New telescopes usually came about either because someone had a bright idea, developed it and then did whatever radio astronomy he could with it, or because someone asked a particular scientific question and then designed an instrument to answer it. These both proved to be very fruitful ways to proceed, especially when the practitioners were as talented and clever as those of the 50's and 60's. The VLA did not come about this way however. Our motives and goals were quite different. We set out from the beginning to build a flexible, general-purpose instrument for a broad scientific purpose, to be used by a lot of people other than, or in addition to, the designers. Many people considered that approach inappropriate, 
unimaginative, undesirable, overly expensive, and unneeded, and we had to cope with those criticisms for 10 or 15 years.

It was decided very early, probably already in 1961 , that we wanted a high resolution, imaging (although we usually said mapping) instrument of the greatest reasonable versatility and of large dynamic range. These goals were set in part because of our concept of what a national center was supposed to do, and in part in response to the limitations of existing instruments. The study of extragalactic radio sources was the primary scientific motivation. We then spent the next 11 or 12 years refining what we meant by high resolution, reasonable versatility, etc., convincing others to agree with us, and designing the specific hardware. For me, the first part, refining the concept, was the more interesting, and the second part, convincing others the hardest, but fortunately there were also people who felt that the specific hardware was also interesting and important.

The work was fairly desultory for the first couple of years, but we did make some progress. In November 1962 I wrote a letter to Geoff Keller at the NSF describing the status of the project. Here, Fig. 2, is the first page of that letter.

As you can see, the specs are still pretty vague, but most of the essential conceptual elements are already here, in the fall of 1962, including aperture synthesis using dishes. The resolution spec went through several iterations as the design proceeded and we became more confident about what was feasible. For example, there was a lot of debate in the 60's about whether atmospheric fluctuations would limit radio resolution, as they did optical, and if so, at what resolution. Note the sensitivity spec. I can't remember what system noise temperatures were back then but they must have been in the hundreds of degrees. The concept described at the bottom of the page was not actually formally adopted until some later time, and various other ideas were also investigated, but apparently we already thought that that concept was the front runner. It is, of course, the aperture super-synthesis concept developed by Martin Ryle.

The letter went on to describe design plans for the next couple of years, and ended with the usual plea for money.

The Green Bank interferometer project was begun in 1963, with the construction of a second, moveable, $85-\mathrm{ft}$ telescope. The third $85-\mathrm{ft}$ and a smaller more distant telescope were added a few years later. These developments were undertaken for two basic reasons: to provide the VLA designers with experience in interferometry and with a test instrument for some of the VLA design questions, for example, atmospheric and instrumental phase stability, and to provide radio astronomers both in and outside of NRAO with some experience in doing science with an interferometer. Up to that time the only interferometer in the US was that at Caltech. We felt that if the VLA was going to depend on interferometry we had better broaden the base of experience. The original 2-element interferometer was developed primarily by Hvatum, Tyler, Hogg, and Wade, with a lot of advice and help from Radhakrishnan and Read at Cal Tech. Others got involved shortly thereafter, especially Clark and Swenson.

In 1964 things began to heat up. The Whitford Committee, formally called the "Panel on Astronomical Facilities," published its report that summer, although its recommendations had been known sometime earlier. The 
November 19, 1962

Dr. Geoffrey Keller

Assistant Director for Mathematical,

Physical, and Engineering Sciences

National Science Foundation

Washington 25, DC

Dear Geoff:

I will try here to give you a little more Information about our proposed antenna project.

We belleve that the next major development the NRAO should undertake is that of an antenna system with a resolution of the order of 10 seconds of arc. The desirable characteristics of such an instrument are roughly as follows:

a) 10 seconds of arc resolution at some centimeter wavelength;

b) ability to map a small region of sky--five to ten minutes of arc in diameter--to the above resolution in a reasonable time. "Reasonable time" is perhaps a few days, but this is a direct case where time required is a function of the money spent;

c) considerable declination coverage;

d) sufficient sensitivity to study several hundred sources;

e) Low side lobe leveis, and no troubles due to grating lobes;

f) ability to change operating wavelength over 10 to 1 range--say $10 \mathrm{~cm}$ to $100 \mathrm{~cm}-$-without great difficulty;

g) antenna system should be expandable, so that by later addition of elements greater resolution could be obtained--or perhaps do same thing by moving the elements.

The most feasible arrangement for such system seems to be an array of moderate size elements, of 80 to $150 . \mathrm{ft}$. dlameter. The number of elements would determine the cost and would also set, along with some other factors. the time required to map a given region or source. It might be desirable that some of the elements be movable, on tracks for example, or that all of the elements be steerable in hour angle in order to utllize the changing orlentation and effective baseline as source is tracked across the sky, or both.

Figure 2. The first page of the letter written to Geoff Keller at the NSF, describing the status of the VLA project.

Panel, with three radio astronomers-Bracewell, Drake and Haddock-among its eight members, had been established in 1962 and had held a big meeting of radio astronomers and others in the fall of 1963. The Panel recommended for radio astronomy the development of ".. . a very powerful high-resolution 
instrument. This is needed particularly for the study of the physics of the bright extragalactic radio sources, and for cosmological studies, and also for other programs in galactic structure and solar-system phenomena. ... (it should) achieve a resolution of less than 10 seconds of arc at centimeter wavelengths." The Panel recommended as a secondary goal a resolution of about 1 arcmin at $21 \mathrm{~cm}$ for hydrogen line studies of galactic structure. It went on to suggest a way to accomplish this, e.g., 100 separate parabolic antennas each 85-ft in diameter, at an estimated (by the Panel) cost of $\$ 40$ million. It also recommended that the instrument be built and operated by the NRAO. This was an important report. It gave, for the first time, some sort of "official" community endorsement of a very sophisticated, powerful, and versatile instrument to be built and operated as a national facility by the NRAO. At NRAO we enthusiastically endorsed the goals and the charge that NRAO do it. We also adopted the price tag. This is important, because you can't design a versatile, more or less general-purpose instrument without imposing some constraints, and cost is certainly one. Adopting the cost, which incidentally in fact translates to the final cost of the VLA when inflation is taken into account, immediately ruled out the committee's proposed array of one hundred 85-ft dishes. The Panel also recommended expansion of the Caltech interferometer by adding four 130-ft dishes and construction of a fully steerable parabola of about 300-ft diameter. The estimated cost of each of these was $\$ 8$ million, and unfortunately neither was done, although one 130 - $\mathrm{ft}$ dish was built.

Some other important things happened in 1964. First, I received a letter from a Caltech graduate student saying that he had been doing hydrogen line interferometry for some time and now would like to come to NRAO to do some single dish hydrogen line work. So Barry Clark came, and to my almost certain knowledge he has yet to do any single dish hydrogen line work at all. So much for good intentions. Then George Swenson announced that he was going to take a leave of absence from Ilinois to come and straighten out the VLA project, or something to that effect. Both of these individuals had key roles in subsequent VLA work, and I can't take any credit for it-they both invited themselves. Barry of course has been a major contributor to just about every NRAO activity since then. George, in addition to his other activities visa-vis the VLA, was also, in my mind, the catalyst that sort of got the VLA design effort into high gear.

And, finally, in 1964 we formally organized a VLA design group. The original group consisted of Clark, Heeschen, Hogg, Hvatum, Tyler, Wade, and Swenson as chairman. Later it included various others, especially Sandy Weinreb when he came to NRAO in 65 , Emil Blum while he was with us, and Bernie Burke, Marshall Cohen, and Bill Erickson from outside NRAO. The group met regularly, at least monthly, from 1964 to well into the construction phase, with the exception of the 1969-71 period when design work was stopped. Table 1 shows the performance goals as they had evolved by 1967. They are not very different from the final specs. There was by no means universal agreement that these were appropriate goals, and there was endless argument about them, both within NRAO and especially in the broader community, right up until construction began.

Many people argued that full sky coverage was unnecessary, or that not so much speed was really needed. A famous optical astronomer whom I won't name even argued during one of the Dicke Committee meetings that one 
arcsec resolution was not really all that necessary or desirable. He made the argument because a competing proposal that he represented was constrained to a site that wouldn't permit such high resolution. Our basic argument in favor of one second resolution was that it was important to have radio resolution comparable to optical. Most of the arguments were aimed at reducing the cost in exchange for a loss in performance. However, the design was finally so well balanced that a change in one parameter would usually impact others as well, and the resultant loss of performance was disproportionately large compared with the cost saving. The two exceptions to this were side lobe level and resolution. The original side lobe goal resulted in an array of 36 antennas. This looked too costly for what we got and the side lobe requirement was reduced from $20 \mathrm{~dB}$ to $15 \mathrm{~dB}$, which led to a 27 -element array. In the case of resolution, scaling down the dimensions of the array would not affect much of anything except resolution and save considerable money. We kept that as a fall-back position for a long time, but fortunately never had to go to it.

\section{TABLE 1}

VLA Performance Goals in 1967

Resolution:

Sensitivity:

Sidelobes:

Field of View:

Sky Coverage:

Speed:

Polarization:

Frequency Range:

Spectroscopy:

Versatility:
1 arcsec at $10-\mathrm{cm}$ wavelength

$0.1-1.0 \mathrm{mJy}$

Similar to those of a parabolic antenna;

first sidelobe at $-20 \mathrm{~dB}$

$1-10$ arcmin

$-20^{\circ}$ to $+90^{\circ}$ declination

$\leq 12^{\mathrm{h}}$ to achieve above goals in a given region of sky

Full Polarization capability

$\geq 3 \mathrm{~cm}$; multiple bands

Yes

Should be as versatile as reasonably possible

These debates, which were carried on in a rather large segment of the radio astronomy community, were very trying for some of us, but in fact they proved quite useful. In the early stages of the design they helped establish the goals, and the continuing debate after performance parameters were set served to provide a more or less continuous review of the entire program. Today, I doubt if any of these parameters would be considered very controversial. Nowadays, for example, the value of speed is pretty well accepted, and we constantly strive for greater speed through faster computers, lower noise systems, multiple feeds, etc. Not so in the 60's. Lots of people wondered loudly and vociferously where all the astronomers were going to come from to use the two or three maps per day the VLA was expected to produce. If it had been known then that the VLA would often produce maps at the rate of one hundred or so per day, we might never have gotten it accepted, just on the grounds that it was far to fast for any conceivable pool of available users.

The VLA concept did not initially enjoy much support, either in the US or in the rest of the world. Many thought it was too expensive and grandiose. In the US some saw it as competition to their own projects or funding. Others thought it was technically infeasible, for one reason or another. A prominent radio astronomer in Europe flatly asserted that it would not be possible to keep 
that much electronics operating reliably. I remember the period from about 1965 to 70 as a time of almost constant attack on the VLA by its critics and competitors. We had a couple of things going for us. One was the enormous increase in power over anything else in existence or planned. This was obvious, very hard to argue against, and was not eroded by the passage of time and other developments. The other was the ultimate thoroughness and excellence of the design, the engineering, and the testing or prototyping of concepts and hardware. Eventually we had a sound answer to just about every possible criticism. Some of this extensive design and testing may have been driven by the criticism, so in that sense the criticism was probably useful, but it almost gave me ulcers at the time.

Well, this is where I will stop my reminiscences. Lots of exciting things were still to come of course, including all of the actual hardware design and the construction. Even for the period I've covered, it is in no sense a comprehensive story. On the contrary, it is incomplete, full of probably very bad selection effects, and maybe even wrong in some respects, so be warned. But it is about as good as I can do without spending an inordinate amount of time with dusty old files, which I don't intend ever to do.

The VLA gave me a lot of headaches for a lot of years. In compensation I had the pleasure and privilege of working with many very talented and dedicated individuals, and now I have the pleasure of seeing the marvelous science coming out of the VLA, so it was well worth it. I wish that all who worked on the VLA could be named, and given the recognition they deserve. I can't do that, but I would like to make special note of those who were heavily involved from the beginning to the end, or almost the beginning to almost the end, namely Barry Clark, Dave Hogg, Bill Horne, Hein Hvatum, Phyllis Jackson, Cam Wade, and Sandy Weinreb. All of these folks were major contributors to the VLA from the early 60's right up through 1980 . Most of those names are familiar to you, but two may not be. Bill Horne was the engineer responsible for, among other things, the antennas and the rail system. He died a few years ago, and we are the poorer for it. Phyllis was, and still is, boss of the NRAO director's office, and about as indispensable as a person can be.

I haven't said much about George Swenson because his main involvement began at about the end of the period I've been discussing, and I haven't said much about Jack Lancaster, because his involvement began long after this period. But now, by way of introduction to their talks, let me relate the following anecdote. At a meeting of the Ad Hoc Advisory Panel in 1976 (Fig. 1) a well-known, non NRAO astronomer now in the Pacific NW told the panel I was a dictator. He added "benevolent" to his description, but in a very soft voice. Well now, I may have wanted to be, and tried hard to be, a dictator, benevolent or otherwise, but I certainly never succeeded. George Swenson and Jack Lancaster are my living proofs of that. Both of them always did exactly what they wanted to do, and both pretty much ignored what I wanted them to do. And to that fortunate circumstance we probably owe much of the success of the VLA. 